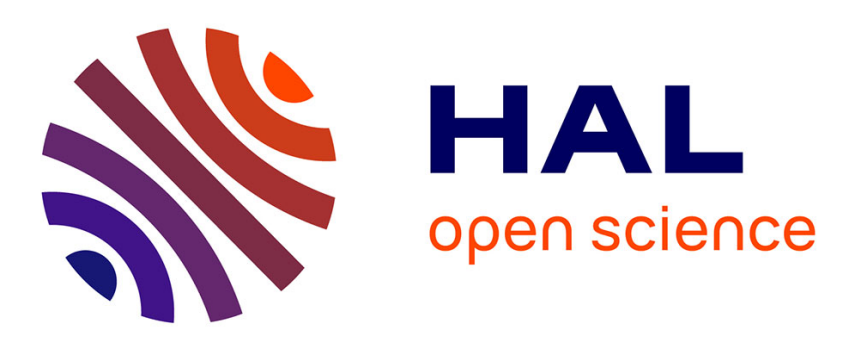

\title{
Periodic motions of a string vibrating against a fixed point-mass obstacle: II
}

\author{
Henri Cabannes
}

\section{To cite this version:}

Henri Cabannes. Periodic motions of a string vibrating against a fixed point-mass obstacle: II. Mathematical Methods in the Applied Sciences, 1984, 6 (1), pp.55-67. 10.1002/mma.1670060105. hal-01527093

\section{HAL Id: hal-01527093 \\ https://hal.science/hal-01527093}

Submitted on 23 May 2017

HAL is a multi-disciplinary open access archive for the deposit and dissemination of scientific research documents, whether they are published or not. The documents may come from teaching and research institutions in France or abroad, or from public or private research centers.
L'archive ouverte pluridisciplinaire HAL, est destinée au dépôt et à la diffusion de documents scientifiques de niveau recherche, publiés ou non, émanant des établissements d'enseignement et de recherche français ou étrangers, des laboratoires publics ou privés. 


\title{
Periodic Motions of a String Vibrating Against a Fixed Point-Mass Obstacle: II
}

\author{
H. Cabannes, Paris
}

\begin{abstract}
A string fixed at both ends $A$ and $B$, can oscillate in a plane in which there is a fixed point obstacle, placed in the middle of the line $A B$. The string is initially at rest with a prescribed shape, symmetric with respect to the normal mid-plane of the segment $A B$. Using results established before [9] we find new periodic motions.
\end{abstract}

\section{Introduction}

During the past few years several works have been devoted to the motion of a string vibrating in the presence of obstacles. Amerio and Prouse [1] are the first to have considered the problem and they have proved, for a straight line obstacle, the global existence of the solution. Schatzman [2] and Bamberger [3] have studied the case of concave obstacles. Cabannes and Haraux $[4,5]$ have studied, for straight line obstacles, the periodic or almost periodic character of the solution. Betro and Gotusso [6] have made numerical computations; Citrini and d'Acunto [7] have considered the shock of two strings. The case of a pointmass obstacle has been studied by Reder [8] and Cabannes [9].

The present work is devoted to the investigation of periodic plane motions of a vibrating-string fixed at both ends $A$ and $B$ in the presence of a point mass obstacle fixed in the middle of $A B$. Initially, the string is at rest with a prescribed shape, which is symmetric about the median line normal to the segment $A B$ : figure 1. The elongation (distance from the equilibrium position) possesses three extrema; one of there, located on the median of $A B$, is above the obstacle, the other two are below the obstacle at positions symmetric with respect to the median. We prove that, when the ratio of corresponding elongations is a rational number, the motion of the string is periodic and we compute the period; when the ratio is irrational the motion of the string is not periodic; it is probably almost periodic but this result still has to be proved.

\section{Statement of the Problem}

The equilibrium position of the string is along the segment $A B$ of the $x$-axis, the points $A$ and $B$ having as abscissae \pm 0.5 ; it can oscillate in a plane 
$x-u$ above a fixed point mass obstacle, placed at the origin. The function $u(x, t)$ which represents the departure from the equilibrium position satisfies the following conditions:

$$
\frac{\partial^{2} u}{\partial t^{2}}-\frac{\partial^{2} u}{\partial x^{2}}=0
$$

(2) $\quad u(0, t)>0$

$$
\begin{aligned}
& u\left( \pm \frac{1}{2}, t\right)=0 \\
& u(x, 0)=\alpha(x), \quad \alpha(0)>0, \quad \alpha\left( \pm \frac{1}{2}\right)=0
\end{aligned}
$$

$$
\frac{\partial u}{\partial t}(x, 0)=\beta(x), \quad \beta\left( \pm \frac{1}{2}\right)=0
$$

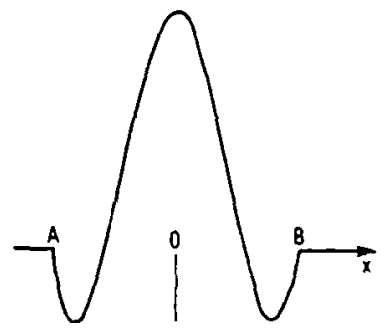

Fig. 1

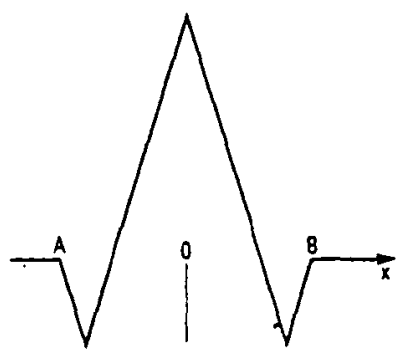

Fig. 2

The contact of the string with the obstacle corresponds to zero values of the function $u(0, t)$ and persists during the intervals of time when the reaction of the obstacle on the string is positive, let (cf. [9] formula (7))

$$
\frac{\partial u}{\partial x}\left(0^{+}, t\right)<\frac{\partial u}{\partial x}\left(0^{-}, t\right) .
$$

At the initial time the string is at rest with a symmetric displacement represented by fig. 1 . One can always assume that the two equal minima have the value -1 ; we denote by $M=\alpha(0)$ the maximum. It is always possible, by means of a change of variable defined by the formula (6), to revert to the case of a string initially at rest in a position $v(x, 0)$, piecewise affine, represented in fig. 2 (cf. [9], theorem 2).

$$
u(x, t)=v\left\{\frac{F(x+t)+F(x-t)}{2}, \frac{F(x+t)-F(x-t)}{2}\right\} .
$$

The new initial shape $v(x, 0)$ is composed of straight line segments, all having the same slope $k=2(M+2)$. We define an odd, increasing function $F(y)$, such that $F(y)-y$ is a periodic function with period 1 , by the relation, 


$$
\alpha(x)=u(x, 0)=v\{F(x), 0\} .
$$

If the motion of the string, initially at rest in the position $v(x, 0)$, is periodic with whole number period $N$, then the motion of the string initially at rest in the position $\left.\alpha(x)=u(x, 0)=v_{\{}^{\prime} F(x), 0\right\}$ is periodic with the same period. Moreover, since the initial data are even functions, the necessary and sufficient condition for the motion of the string to be periodic, is that the motion of the middle of the string is periodic. The motion of the string and the motion of the mid-point therefore have the same periods (cf. [9], theorem 3).

We adopt henceforth for the function $\alpha(x)$ the function represented in figure 2 .

$$
\alpha(x)=M-k x \quad \text { for } 0 \leqslant x \leqslant \frac{M+1}{k}
$$

$$
\begin{aligned}
& \alpha(x)=k\left(x-\frac{1}{2}\right) \quad \text { for } \frac{M+1}{k} \leqslant x \leqslant \frac{1}{2} \\
& \alpha(-x)=\alpha(x)
\end{aligned}
$$

$$
\begin{aligned}
& \alpha(1-x)=-\alpha(x) \quad k=2(M+2) . \\
& \alpha(x+2)=\alpha(x)
\end{aligned}
$$

\section{Motion of the Middle of the String}

The motion of the middle of the string $u(0, t)$ can be studied without the knowledge of the complete motion of the string. Before the first contact, we have the free oscillation

$$
u(0, t)=f^{0}(t)=\alpha(t) .
$$

The first contact begins at the time $C_{0}$, which is the smallest positive root of the equation $f^{0}(t)=0$; we therefore have:

$$
C_{0}=\frac{M}{k} \text {. }
$$

This first contact ends at the time $D_{0}$ when the reaction vanishes; $D_{0}$ is the smallest root greater than $C_{0}$ of the equation $\left(\mathrm{d} f^{0} / \mathrm{d} t\right)=0$; we have therefore

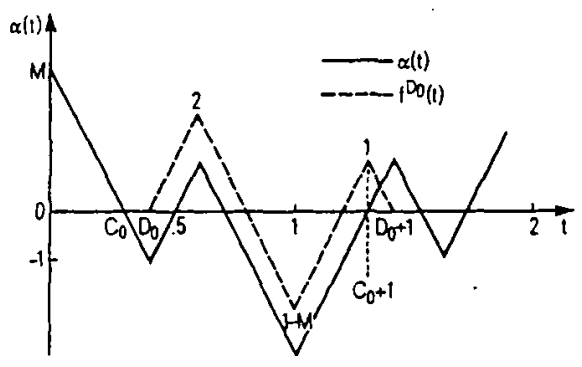

Fig. 3 


$$
D_{0}=\frac{1+M}{k} .
$$

After time $D_{0}$ we have a new free oscillation.

In general after the time $D_{n}$ which follows the end of a contact we have a new free oscillation in which the motion of the middle of the string $f^{D_{n}}(t)$ is defined (cf. [9], theorem 5) by the relations:

$$
\begin{aligned}
& f^{D_{n}(t)}=f^{D_{n-1}(t)-f^{D_{n-1}}\left(D_{n}\right)} \quad \text { for } D_{n} \leqslant t \leqslant 1+C_{n}
\end{aligned}
$$

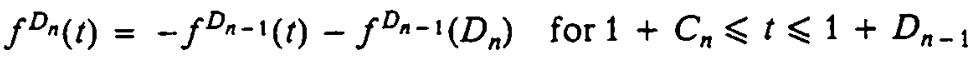

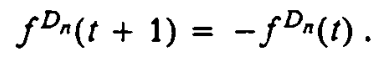

The contact which follows the detachement at the time $D_{n}$ appears at the time $t=C_{n+1}$ which is the smallest root greater than $D_{n}$ of the equation $f^{D_{n}(t)}=0$, and this contact ends at the time $t=D_{n+1}$, which is the smallest root greater

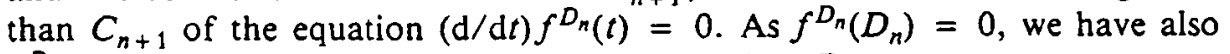
$f^{D_{n}}\left(1+D_{n}\right)=0$, and $C_{n+1}$ is at most equal to $1+D_{n}$.

The first complete free oscillation, that which occurs after time $D_{0}$, is defined by the functions

$$
\begin{array}{ll}
f^{D_{0}}(t)=\alpha(t)+1 & \text { for } D_{0} \leqslant t \leqslant 1+C_{0} \\
f^{D_{0}}(t)=-\alpha(t)+1 & \text { for } 1+C_{0} \leqslant t \leqslant 1+D_{0}
\end{array}
$$

We deduce that in the interval $D_{0} \leqslant t \leqslant 1+D_{0}$, the function $f^{D_{0}}(t)$ has a first maximum $M_{1}^{0}=2$, a minimum $m^{0}=1-M$ and a second maximum $M_{2}^{0}=1$ : Fig. 3.

We assume that the free oscillation, which starts at the time $D_{n}$, has, for $D_{n} \leqslant t$ $\leqslant 1+D_{n}$, a first maximum $M_{1}^{n}$, a minimum $m^{n}$ and a second maximum $M_{2}^{n}$. The formulae (13) prove that the same situation is valid for the free oscillation which starts at the time $D_{n+1}$. As this is true for $n=0$, this is always true and from formulae (13) we have:

If $m^{n} \geqslant$

(Fig. 4)

$$
\begin{array}{ll}
M_{1}^{n+1}=M_{1}^{n}-m^{n}, & C_{n+1}=1+D_{n} \\
m^{n+1}=M_{1}^{n}-M_{2}^{n}, & D_{n+1}=\frac{M_{1}^{n}}{k}+C_{n+1} \\
M_{2}^{n+1}=M_{1}^{n}, & D_{n} D_{n+1}=1+\frac{M_{1}^{n}}{k} \\
M^{n+1}=M_{2}^{n}-m^{n}, & C_{n+1}=\frac{2 M_{1}^{n}}{k}+D_{n}
\end{array}
$$

If $m^{n}<0$

(Fig. 5)

$$
\begin{array}{ll}
m^{n+1}=-M_{1}^{n}-m^{n}, & D_{n+1}=-\frac{m^{n}}{k}+C_{n+1} \\
M^{n+1}=-m^{n} & D_{n} D_{n+1}=\frac{2 M_{1}^{n}-m^{n}}{k} .
\end{array}
$$


We note that the sum $M_{1}^{n}-m^{n}+M_{2}^{n}$ is independent of the index $n$ and so has the value $M+2$.

Moreover it follows from formulae (16) and (17) that if $M$ is a rational number ( $M=p / q, p$ and $q$ denoting two mutually prime numbers; $p$ positive or zero, $q$ strictly positive), $M_{1}^{n}$ and $M_{2}^{n}$ have the following form

$$
M_{1}^{n}=\frac{p_{1}^{n}}{q}, \quad M_{2}^{n}=\frac{p_{2}^{n}}{q} .
$$

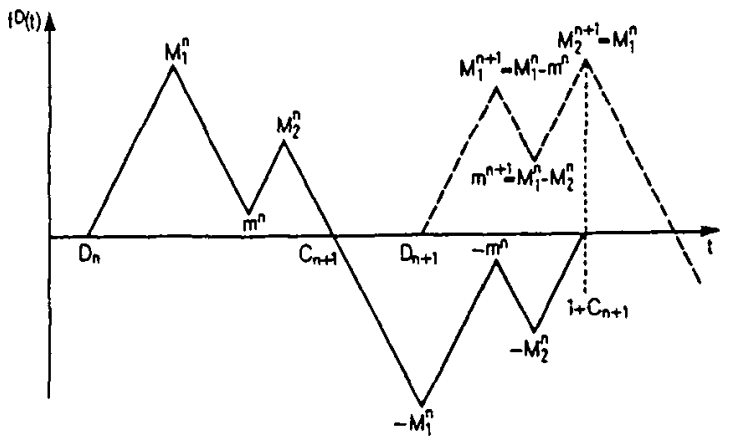

Fig. 4

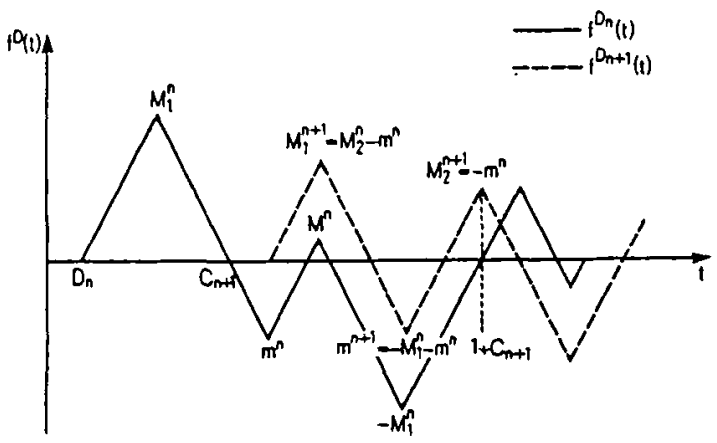

Fig. 5

The quantities $p_{1}^{n}$ and $p_{2}^{n}$ are integers which can have only a finite number of values.

To prove this result we assume first that we have $M<2$, and prove that the following inequalities are satisfied for all values of $n$ :

$$
M \leqslant M_{1}^{n} \leqslant 2, \quad 0 \leqslant M_{2}^{n} \leqslant 2 .
$$

The property being true for $n=0$, it is sufficient to deduce its validity for $n+1$ from that for $n$. This follows from relations (16) if we have $m^{n} \geqslant 0$, from relations (17) if we have $m^{n}<0$

$$
\begin{array}{ll}
\text { Ist case } m^{n} \geqslant 0 \quad & M_{1}^{n+1}=M_{1}^{n}-m^{n} \leqslant M_{1}^{n} \leqslant 2 \\
& M_{1}^{n+1}=M+2-M_{2}^{n} \geqslant M \\
& M_{2}^{n+1}=M_{1}^{n} \text { then } 0 \leqslant M_{2}^{n+1} \leqslant 2
\end{array}
$$


2nd case $m^{n}<0 \quad M^{n+1}=M_{2}^{n}-m^{n}=2+M-M_{1}^{n}$

$$
\begin{aligned}
& M_{1}^{n} \text { varying from } M \text { to } 2, \quad M_{1}^{n+1} \text { varies from } M \text { to } 2 \\
& M_{2}^{n+1}=-m^{n}>0 \\
& M_{2}^{n+1}=2+M-M_{1}^{n} \leqslant 2
\end{aligned}
$$

We assume then that we have $M>2$, and we prove that the following inequalities are satisfied for all values of $n$

$$
\text { (19-b) } 2 \leqslant M_{1}^{n} \leqslant M, \quad 0 \leqslant M_{2}^{n} \leqslant M \text {. }
$$

The property is true for $n=0$. We then have by induction

$$
\begin{array}{ll}
\text { Ist case } m^{n} \geqslant 0 \quad & M_{1}^{n+1}=M_{1}^{n}-m^{n} \leqslant M_{1}^{n} \leqslant M \\
& M_{1}^{n+1}=M+2-M_{2}^{n} \geqslant 2 \\
& M_{2}^{n+1}=M_{1}^{n+1} \text { then } 0 \leqslant M_{2}^{n+1} \leqslant M . \\
\text { 2nd case } m^{n}<0 \quad & M_{1}^{n+1}=M_{2}^{n}-m^{n}=2+M-M_{1}^{n} \\
& M_{1}^{n} \text { varying from } 2 \text { to } M, M_{1}^{n+1} \text { varies from } M \text { to } 2 \\
& M_{2}^{n+1}=-m^{n}>0 \\
& M_{2}^{n+1}=2+M-M_{1}^{n}-M_{2}^{n} \leqslant M .
\end{array}
$$

Assembling all these results, we have:

$$
\inf (p, 2 q) \leqslant p_{1}^{n} \leqslant \sup (p, 2 q), \quad 0 \leqslant p_{2}^{n} \leqslant \sup (p, 2 q) .
$$

The pair $\left(p_{1}^{n}, p_{2}^{n}\right)$ can only have a finite number of values and after a finite number of contacts and free oscillation we obtain for the couple $M_{1}^{n}, M_{2}^{n}$ values already found:

$$
\begin{aligned}
& M_{1}^{n+m}=M_{1}^{n}, \quad M_{2}^{n+m}=M_{2}^{n}, \quad \text { from which we deduce } \\
& u\left(0, t+D_{n+m}\right)=u\left(0, t+D_{n}\right)
\end{aligned}
$$

which proves that the motion is periodic with the period $T=D_{n} D_{n+m}$, a period which is finite because $D_{i} D_{i+1}$ is always less than $3 / 2$, as a result of various inequalities, established previously.

\section{Calculation of the Period for $M=p / q>2$}

To compute the period of motion when $M$ is rational, we begin by calculating the quantities $M_{1}^{i}, m^{i}$ and $M_{2}^{i}$. To do this we start from the case $i=0$ :

$$
M_{1}^{0}=2, \quad m^{0}=1-M, \quad M_{2}^{0}=1
$$

and we reason by induction, using either formulae (16) or formulae (17).

We put $M=p / q, p$ and $q$ being mutually prime integers, $p$ positive or zero, $q$ strictly positive. We first assume that $p>2 q$, and we put ${ }^{1}$ )

5) The notation $[x]$ denotes the greatest integer smaller or equal to $x$, that means the integral part of $x$. 


$$
\sigma_{j}=\left[\frac{(j+1) M-1}{2}\right] \quad(j=0,1,2, \ldots) .
$$

In particular $\sigma_{-1}=-1, \sigma_{2 q-1}=p-1$. From the relation (22) we deduce

$$
\begin{array}{ll}
\text { (23-1) } \sigma_{j}<\frac{(j+1) M-1}{2} & \text { if } \frac{(j+1) M-1}{2} \text { is not an integer } \\
\text { (23-2) } \sigma_{j}=\frac{(j+1) M-1}{2} & \text { if } \frac{(j+1) M-1}{2} \text { is an integer. }
\end{array}
$$

If $p$ is even, $q$ is odd and the inequality (23-1) is always satisfied. If $p$ is odd the equality (23-2) is satisfied for $(j+1) p=(2 m+1) q ; m$ is an integer and $2 m+1$ an odd multiple of $p$. We have therefore $m=(p-1) / 2$ and $j=q-1$, or $m=(3 p-1) / 2$ and $j=3 q-1, \ldots$ Also when the index $j$ varies from 0 to $2 q$ the inequality (23-1) is always satisfied except if $p$ is odd and if $j=q-1$. The equality is then satisfied and we have

$$
\sigma_{q-1}=\frac{q M-1}{2}=\frac{p-1}{2} .
$$

We assume first that $p$ is an odd integer. We denote by $s_{j}^{1}, s_{j}^{2} \ldots$ the set of integers greater than $\sigma_{j-1}$, less or equal to $\sigma_{j} ; s_{j}^{1}=1+\sigma_{j-1}, \ldots$

$$
\sigma_{j-1}<s_{j} \leqslant \sigma_{j} \quad(j=0,1, \ldots 2 q)
$$

$j$ varies from 0 to $2 q$ and the absence of the upper index in the inequalities (24) means that $s_{j}$ represents any of the integers satisfying those inequalities. From relations (23) and (24) we deduce:

$$
\begin{array}{ll}
s_{j} \leqslant \sigma_{j}<\frac{(j+1) M-1}{2}<\sigma_{j}+1 & \text { for } j \neq q-1 \\
s_{j} \leqslant \frac{(j+1) M-3}{2} & \text { for } s_{j} \neq \sigma_{j} \\
\sigma_{j-1} \leqslant \frac{j M-1}{2}<s_{j} . &
\end{array}
$$

These various inequalities enable us to construct the table of the values of $M_{1}^{i}, m^{i}$ and $M_{2}^{i}$. For $s_{j} \neq \sigma_{j}$ this table $\mathrm{I}-\mathrm{a}$ is the following:

\begin{tabular}{l|lll}
$i$ & $M_{1}^{i}$ & $m^{i}$ & $M_{2}^{i}$ \\
\hline $3 s_{j}$ & 2 & $1+2 s_{j}-(j+1) M<0$ & $M+m^{i}$ \\
$3 s_{j}+1$ & $M$ & $(j+1) M-\left(3+2 s_{j}\right) \geqslant 0$ & $2+m^{i}$ \\
$3 s_{j}+2$ & $2+m^{i}$ & $1+2 s_{j}-j M>0$ & $M$
\end{tabular}

The value of $m^{i}$ on the first line is negative by virtue of inequalities (25); that on the second line is positive or zero by virtue of the inequality (26), and that on the 
third line is positive by virtue of the inequality (27). The first line is obtained for $s_{0}=0$, the two following lines are obtained by application either of relations (16), or of relations (17). Finally, if we apply the relations (16) to the third line we find again the first line in which $s_{j}$ is replaced by $1+s_{j}$.

For $s_{j}=\sigma_{j}$ with $j \neq q-1$ table I-a must be replaced by table I-b, which follows,

\begin{tabular}{l|lll}
$i$ & $M_{1}^{i}$ & $m^{i}$ & $M_{2}^{i}$ \\
\hline $3 \sigma_{j}$ & 2 & $1+2 \sigma_{j}-(j+1) M<0$ & $M+m^{i}$ \\
$3 \sigma_{j}+1$ & $M$ & $(j+1) M-\left(3+2 \sigma_{j}\right)<0$ & $2+m^{i}$
\end{tabular}

The value of $m^{i}$ on the second line is negative by virtue of the last of inequalities (25). If we apply the relations (17) to the second line we find again the first line of table I-a in which $j$ is replaced by $j+1$ and $s_{j}$ by $s_{j+1}=1+\sigma_{j}$.

For $s_{j}=\sigma_{q-1}$ we must replace table I-b by the following table I-c:

\begin{tabular}{l|lll}
$i$ & $M_{1}^{i}$ & $m^{i}$ & $M_{2}^{i}$ \\
\hline $3 \sigma_{q-1}$ & 2 & 0 & $M$
\end{tabular}

By application of relations (16) we find again the first line of table I-a, in which $j=q$ and $s_{j}=s_{q}=1+\sigma_{q-1}$.

We can collect these conclusions together by saying that, for $j$ varying from 0 to $2 q$, table I-a is always valid on condition that we cancel

$$
\begin{aligned}
& \text { all the lines of rank } 3 \sigma_{j}+2 \\
& \text { and the line of rank } 3 \sigma_{q-1}+1=\frac{3 p-1}{2} .
\end{aligned}
$$

For $j=2 q$ the first term of the sequence $s_{j}=s_{2 q}$ is $1+\sigma_{2 q-1}=p$. For $j=2 q$ and $s_{2 q}=p, i=3 s_{2 q}=3 p$, we obtain as first line of table I-a:

$$
M_{1}^{3 p}=2, \quad m^{3 p}=1-M, \quad M_{2}^{3 p}=1 .
$$

We have thus shown that the motion is periodic, but in addition, we have determined the value of the period $T=D_{0} D_{3 p}$. To calculate this period it is

\begin{tabular}{|c|c|c|}
\hline$i$ & $D_{i} D_{i+1}$ & with $k=2(M+2)$ \\
\hline $3 s_{j}$ & $\frac{(j+1) M+3-2 s_{j}}{k}$ & \\
\hline $3 s_{j}+1$ & $1+\frac{M}{k}$ & $3+\frac{2}{k}$ \\
\hline $3 s_{j}+2$ & $1+\frac{3+2 s_{j}-j M}{k}$ & \\
\hline
\end{tabular}
enough to calculate the length of intervals $D_{i} D_{i+1}$; this length is given in the following table: 


$\left.\begin{array}{l|ll}i & D_{i} D_{i+1} & \text { with } k=2(M+2) \\ \hline 3 \sigma_{j} & \frac{(j+1) M+3-2 \sigma_{j}}{k} \\ 3 \sigma_{j}+1 & \frac{3+\sigma_{j}-(j-1) M}{k}\end{array}\right] \begin{aligned} & 1+\frac{2}{k} \\ & 3 \sigma_{q-1}\end{aligned}$

The number of groups of intervals of the first type is $\left(\sigma_{2 q-1}-2 q\right)+1=p$ $-2 q$. The number of groups of intervals of the second type is $2 q-1$ (from $\sigma_{0}$ to $\sigma_{2 q-1}$, except $\left.\sigma_{q-1}\right)$. There is one interval of the third type. We have therefore:

$$
D_{0} D_{3 p}=(p-2 q)\left(3+\frac{2}{k}\right)+2 q\left(1+\frac{2}{k}\right)
$$

(28) $T=D_{0} D_{3 p}=\frac{3 p^{2}+3 p q-8 q^{2}}{p+2 q}$.

When $p$ is even the equality (23-2) is never satisfied and the table I-a is valid with the suppression only of the lines of rank $3 \sigma_{j}+2$. For $j=q$ and $s_{j}=s_{q}=1$ $+\sigma_{q-1}=p / 2$, we obtain for the first line of the table I-a $(i=3 p / 2)$ :

$$
M_{1}^{3 p / 2}=2, \quad m^{3 p / 2}=1-M, \quad M_{2}^{3 p / 2}=1 .
$$

The period of the motion is then $D_{0} D_{3 p / 2}$ or $D_{3 p / 2} D_{3 p}$, that is, half of the value given by the formula (26).

5 Calculation of the Period for $M=p / q<2$

When $p$ is less than $2 q$, we put:

$$
\sigma_{j}=\left[\frac{2 j+1}{M}\right] \quad(j=0,1, \ldots) \text {. }
$$

In particular we have $\sigma_{-1}<-1, \sigma_{p-1}=\left[2 q-\frac{q}{p}\right], \sigma_{p}=\left[2 q+\frac{q}{p}\right]$. From
the relation (29) we deduce:

$$
\begin{array}{ll}
\text { (30-1) } \sigma_{j}<\frac{2 j+1}{M} & \text { if } \frac{2 j+1}{M} \text { is not an integer } \\
\text { (30-2) } \sigma_{j}=\frac{2 j+1}{M} & \text { if } \frac{2 j+1}{M} \text { is an integer }
\end{array}
$$

If $p$ is even, $q$ is odd and the inequality (30-1) is always satisfied. If $p$ is odd the equality (30-2) is satisfied for $(2 j+1) q=m p ; m$ is integer and multiple of $q$. 
We have therefore $m=q$ and $j=(p-1) / 2$, or $m=3 q$ and $j=(3 p-1) / 2$, .... Also when the index $j$ varies from 0 to $p$ the inequality (30-1) is always satisfied, except if $p$ is odd and $j=(p-1) / 2$. The equality (30-2) is then satisfied and we have $\sigma_{(p-1) / 2}=q$.

We assume first that $p$ is odd. We denote by $s_{j}^{1}, s_{j}^{2} \ldots$ the set of integers greater than $\sigma_{j-1}$, less than or equal to $\sigma_{j} ; s_{j}^{1}=1+\sigma_{j-1}, \ldots$

$$
\sigma_{j-1}<s_{j} \leqslant \sigma_{j} \quad(j=0, \ldots p)
$$

$j$ varies from 0 to $p$, and the absence of the upper index in the inequalities (31) means that $s_{j}$ represents any one of the integers satisfying those inequalities. From relations (30) and (31) we deduce:

$$
\begin{array}{ll}
s_{j} \leqslant \sigma_{j}<\frac{1-2 j}{M}<1+\sigma_{j} & \text { for } j \neq \frac{p-1}{2} \\
s_{j} \leqslant \frac{2 j-1}{M} & \text { for } s_{j} \neq \sigma_{j} \\
\sigma_{j-1} \leqslant \frac{2 j+1}{M}<s_{j} . &
\end{array}
$$

These different inequalities allow us to construct the table of values of $M_{1}^{i}, m^{i}$ and $M_{2}^{i}$. For $s_{j} \neq \sigma_{j}$ this table II-a is the following:

\begin{tabular}{l|lll}
$i$ & $M_{1}^{i}$ & $m^{i}$ & $M_{2}^{i}$ \\
\hline $3 s_{j}-1$ & $M$ & $s_{j} M-(1+2 j)<0$ & $2+m^{i}$ \\
$3 s_{j}$ & 2 & $1+2 j-\left(1+s_{j}\right) M \geqslant 0$ & $M+m^{i}$ \\
$3 s_{j}+1$ & $M+m^{i}$ & $s_{j} M-(2 j-1)>0$ & 2
\end{tabular}

The value of $m^{i}$ on the first line is negative by virtue of inequalities (32); that on the second line is positive or zero by virtue of inequality (33) which can be written $2 j-1>M s_{j}$, which implies $2 j+1>M\left(1+s_{j}\right)$ because $M$ is less than 2 . The value of $m^{i}$ on the third line is positive by virtue of inequality (34). The second line is obtained from the values for $s_{0}=0$, the third is obtained by applying the formulae (16); and if we then applied the formulae (16) to this third line, we obtain the first line in which $s_{j}$ is replaced by $1+s_{j}$. The formulae (17) allows us then to obtain the second line.

For $s_{j}=\sigma_{j}$ with $j \neq(p-1) / 2$ the table II-a must be replaced by the following table II-b:

\begin{tabular}{l|lll}
$i$ & $M_{1}^{i}$ & $m^{i}$ & $M_{2}^{i}$ \\
\hline $3 \sigma_{j}-1$ & $M$ & $\sigma_{j} M-(1+2 j)<0$ & $2+m^{i}$ \\
$3 \sigma_{j}$ & 2 & $1+2 j-\left(1+\sigma_{j}\right) M<0$ & $M+m^{i}$
\end{tabular}

The value of $m^{i}$ on the second line is negative by virtue of the last inequality (32). If we applied the relations (17) to this second line we find the first line of the table II-a in which $j$ is replaced by $j+1$ and $s_{j}$ has $s_{j+1}=1+\sigma_{j}$. 
For $s_{j}=\sigma_{(p-1) / 2}=q$ we must replace the table II-b by the following table II-c:

\begin{tabular}{l|lll}
$i$ & $M_{1}^{i}$ & $m^{i}$ & $M_{2}^{i}$ \\
\hline $3 \sigma_{(p-1) / 2}$ & $M$ & 0 & 2
\end{tabular}

By application of relations (16) we find again the first line of the table II-a, in which $j=(p+1) / 2$ and $s_{j}=\sigma_{(p+1) / 2}=1+\sigma_{(p-1) / 2}$.

We can summarize these conclusions by saying that, if $j$ varies from 1 to $p$, the table II-a is always valid under the condition to cancel:

all the lines of rank $3 \sigma_{j}+1$

and the line or rank $3 \sigma_{(p-1) / 2}=3 q$.

For $j=p$ one of the possible values of the set $s_{p}$ is $s_{p}=2 q$, since we have $\sigma_{p-1}$ $=[2 q-(q / p)]<2 q \leqslant[2 q+(p / q)]=\sigma_{p}$.

For $j=p, s_{p}=2 q$ and $i=3 s_{p}=6 q$ we obtain from the second line of table II-a, or of table II-b:

$$
M_{1}^{6 q}=2, \quad m^{6 q}=1-M, \quad M_{2}^{6 q}=1 .
$$

We verify also that the motion is periodic and we obtain the value of the period $T=D_{0} D_{6 q}$. To compute this period it is sufficient to compute the length of the intervals $D_{i} D_{i+1}$ : this length is given in the following table:

\begin{tabular}{l|ll}
$i$ & $D_{i} D_{i+1}$ & with $k=2(M+2)$ \\
\hline $3 s_{j}-1$ & $\frac{1+2 j-\left(s_{j}-2\right) M}{k}$ & $3+\frac{M}{k}$ \\
$3 s_{j}$ & $1+\frac{2}{k}$ & $3+\frac{\left(1+s_{j}\right)-2(j-1)}{k}$ \\
$3 s_{j}+1$ & $1+\frac{1+2 j-\left(\sigma_{j}-2\right) M}{k}$ \\
$3 \sigma_{j}-1$ & $\frac{3-2 j+\left(1+\sigma_{j}\right)}{k}$ & $1+\frac{M}{k}$ \\
$3 \sigma_{j}$ & $\frac{M}{k}$ & $1+\frac{M}{k}$
\end{tabular}

The number of groups of intervals of the first type is $\left(\sigma_{p-1}-p\right)+\left(2 q-\sigma_{p-1}\right)$ $=2 q-p$. The number of groups of intervals of the second type is $p-1$ (from $\sigma_{0}$ to $\sigma_{p-1}$ except $\sigma_{(p-1) / 2}$. There is one interval of the third type. We have also

$$
D_{0} D_{6 q}=(2 q-p)\left(3+\frac{M}{k}\right)+p\left(1+\frac{M}{k}\right)
$$




$$
T=D_{0} D_{6 q}=\frac{12 q^{2}+3 p q-2 p^{2}}{p+2 q} .
$$

When $p$ is even the equality (30-2) is never satisfied and the table II-a is valid by cancelling only the lines of rank $3 \sigma_{j}$. We have

$$
\sigma_{(p / 2)-1}=\left[q-\frac{q}{p}\right]<q<\left[q+\frac{q}{p}\right]=\sigma_{p / 2}
$$

and for $j=p / 2$ the set $s_{j}$ contains the value $s_{j}=s_{p / 2}=q$. If $M<1$ we have $q=s_{p / 2}<\sigma_{p / 2}$, if $M>1$ we have $q=s_{p / 2}=\sigma_{p / 2}$. So, using the tables II-a, or the tables II-b we obtain:

$$
M_{1}^{3 p}=2, \quad m^{3 p}=1-M, \quad M_{2}^{3 p}=1 .
$$

The period of motion is $D_{0} D_{(3 p / 2)}$ or $D_{3 p / 2} D_{3 p}$, that means the half of the value given by the formula (35).

\section{Study of the Cases $M=2$ and $M$ Irrational}

When the ratio $M$ of initial maxima displacements (transverse departure from equilibrium) has the value 2, we have for all $i$ :

$$
M_{1}^{i}=2, \quad m^{i}=-1, \quad M_{2}^{i}=1 .
$$

The period of the motion is $T=D_{i} D_{i+1}=5 / 8$. This value is equal to the fourth of the value given by any one of the formulae (28) or (35).

We assume now that $M$ is irrational. To fix ideas, we assume it is greater than 2; the case where it would be smaller than 2 is similar. The equality (23-2) is never satisfied and the tables I-a and I-b are always valid. If the motion were periodic we would find the value $M_{1}=2$ at an infinite number of times; this is possible only if $i=3 s_{j}$ or $i=3 \sigma_{j}$. The corresponding $m_{i}$ are of the form $\lambda_{i}+\mu_{i} M$ where $\lambda_{i}$ and $\mu_{i}$ are integers; the $\lambda_{i}$ are all different; they are the consecutive odd integers. Therefore if the motion were periodic we would have $\lambda_{i}+\mu_{i} M=\lambda_{i+m}$ $+\mu_{i+m} M$, which means that $M$ is rational.

The motion of the string is therefore not periodic when $M$ is irrational. As we have said in the introduction it is probable that the motion is almost periodic. We hope that a reader will be able to prove this conjecture.

\section{Conclusion}

We have studied the case of a string initially at rest in a position represented by Fig. 2. The maxima displacements are reached for $x=0$ and for $x= \pm \alpha$; their ratio $M=u(0,0) / u(0, \alpha)$ has been assumed rational: $M=p / q$. We have then proved that the motion is periodic and we have computed the periods: formulae (28) and (35). The transformation (6), which conserves the integral periods, allows us to pass from the case of figure 2, to the case of Fig. 1, 
the ratio of maxima displacements being the same. Also when the string is initially at rest in a position represented on the Fig. 1 , with $M=p / q$ ( $p$ and $q$ integers), the motion of the string is periodic and the period has as value:

$$
\begin{array}{ll}
T=12 q^{2}+3 p q-2 p^{2} & \text { if } p \leqslant 2 q \\
T=3 p^{2}+3 p q-8 q^{2} & \text { if } p \geqslant 2 q .
\end{array}
$$

When $M$ is irrational, the motion is not periodic.

\section{References}

[1] A merio, L.; Prouse, G.: Study of the motion of a string vibrating against an obstacle. R. C. di Mat. 8 (1975) $563-585$

[2] Schatzman, M.: An hyperbolic problem of second order with unilateral constraints: the vibrating string with a concave obstacle. Publ. Universite Paris VI, $\mathrm{n}^{\circ} 78031$

[3] Bamberger, A.: Thèse de doctorat. Universite Paris VI, 1979

[4] Cabannes, H.; Haraux, A.: Mouvements presque-périodiques d'une corde virbrante en présence d'un obstacle fixe, rectiligne ou ponctuel. Int. J. Non-Linear Mech. 16 (1981) $449-458$

[5] Haraux, A.; Cabannes, H.: Almost periodic motion of a string vibrating against a straigth fixed obstacle. Nonlinear Anal., Theory, Methods \& Applications 7 (1983) 129-141

[6] Betro, B.; Gotusso, L.: Calcolo numerico di una corda vibrante contra un obstacolo. Instituto per le applicazioni del calcolo "Mauro Picone" - Publ. serie III, $\pi^{\circ} 114,1977$

[7] Citrini, C.; d'Acunto, B.: Sur le choc de deux cordes. C. R. Acad. Sci. Paris 289 A (1979) 5-7

[8] Reder, C.: Etude qualitative d'un problème hyperbolique avec contrainte unilatérale. Thèse de 3ème cycle, Université de Bordeaux, 1979

[9] Cabannes, H.: Mouvements périodiques d'une corde vibrante en présence d'un obstacle ponctuel. J. de Méc. 20 (1981) 1-18

Henri Cabannes

Laboratoire de Mécanique Théorique

associé au C.N.R.S.

Université Pierre et Marie Curie

4 place Jussieu

F-75005 Paris 